\title{
Rational Critique of the Genome Editing Infants Event
}

\author{
Zhai Jiancai, Mei Lin, Song Tonglin, Zhang Xiaolin, Huang Jun \\ Evaluation and Examination Center, Army Medical University, Chongqing, China
}

\section{Email address:}

Zhaimit@qq.com (Zhai Jiancai)

\section{To cite this article:}

Zhai Jiancai, Mei Lin, Song Tonglin, Zhang Xiaolin, Huang Jun. Rational Critique of the Genome Editing Infants Event. Science Discovery. Vol. 7, No. 1, 2019, pp. 22-25. doi: 10.11648/j.sd.20190701.16

Received: January 17, 2019; Accepted: March 20, 2019; Published: April 28, 2019

\begin{abstract}
Genome editing is a new technology in BME and will be widely used in medicine. The birth of genome-edited babies has resulted in serious adverse consequences, for disobeying the international consensus that gene-editing embryos should not be used for pregnancy purposes, disregarding the existing laws and ethical norms of our country, ignoring the scientific warning of dangers of the immature medical practice and betraying the medical spirit and professional integrity. The rational criticism of this incident makes all scientific and medical communities soberly realize that medicine is a weapon created by human civilization for the benefit, which has both material function and spiritual value. Any medical activity could not betray its spiritual value, that is, medical humanistic value. Medical laws and ethics can support and regulate medical activities, guiding to selectively perform the role of science and technology in medical practice, and enable medicine to completely serve the human rights and health protection, rather than to guide gaining benefits for small interest groups. The rational criticism of events must have critical rationality, especially the proper grasp and implementation of the moral norms towards the people concerned with events.
\end{abstract}

Keywords: Genome Editing, GE Infants Event, Rational Critique, Medical Ethics, Medical Humanism

\section{基因组编辑婴儿事件的理性批判}

\section{翟建才，梅林，宋桐林，张晓琳，黄俊}

陆军军医大学评价与考试中心, 重庆, 中国

\section{邮箱}

zhaimit $a$.qq.com（翟建才）

摘要：基因组编辑是生物医学工程一项新技术，必将获得广泛的医学应用。但基因组编辑婴儿出生事件公然违背基因 编辑胚胎不得用于妊娠目的国际共识,置我国现行法律和伦理规范于不顾, 置医学实践危险性的科学预警于不顾, 背叛 医学精神和职业操守, 造成了严重的不良后果。对此事件的理性批判使科学界和广大医学工作者清醒地认识到: 医学 是人类文明造就的一件为人类谋福祉的武器, 既具有物质功能, 又富含精神价值。任何医学活动都不能忽略其精神价 值, 即医学人文价值。医学法律法规和伦理学对医学活动具有支持和调控作用, 能够在医学实践中起到有选择地发挥 科学技术作用的导向作用, 让科学技术向真正服务于人类的人权和健康保障, 而不是为少数利益集团牟利的方向发展。 对事件的理性批判必须具有批判的理性，特别是需要对事件相关人的道德准则的适度把握和实施。

关键词：基因组编辑，婴儿事件，理性批判，医学伦理学，医学人文 


\section{1. 前言}

在香港第二届国际人类基因组编辑峰会召开前一天, 媒 体公开宣布一对名为露露和娜娜的基因编辑婴儿已于 2018 年11月在中国健康诞生。这对双胞胎的一个基因经过修改, 使她们出生后即能天然抵抗艾滋病。消息的公布立即引起国 内外社会和学术界的极大关注。不过科学界并没有像发布者 所期待的对此项“中国在基因编辑技术用于疾病的预防领域 实现历史性突破”的庆贺和赞扬, 更多的却是对此事件背离 生物医学研究的医学精神以及对法律和伦理规范的挑战所 带来的理性批判和科学反思。国家自然科学基金委于11月 28 日及时地对此事件发布公开信，表示“对其作法给人类可能 造成的后果表示极度担忧, 对其严重违反我国现行法律法规 和科学伦理的不负责任行为予以严厉谴责”。

\section{2. 事件对医学精神的背叛}

现代著名的医学史学者西格里斯特(Henry E. Sigerist) 提出[1], “医学充分参与了自然科学的伟大崛起, 借此, 它变得远比过去更加有效。在新的医学科学中, 文明已经 铸就了一件武器, 可以用来努力把人从疾病的古老束缚中 解放出来”。同时又指出, “文明是一个非常复杂的现象, 它既有物质的方面, 也有精神的方面。文明需要对所有精 神价值的养育, 正是这些精神价值, 使生活成为真正的人 的生活, 并因此是值得过的生活。医学能扮演的角色很有 限, 但医学很重要, 因为它对人类福祉做出了极大的贡献, 帮助创造了文化发展的条件”。

这就是说, 从历史的视域来看, 医学是一件随着社会 而发展的科学和文明的武器。它既具有物质功能, 又蕴含 精神价值。正是医学的精神价值而非其工具效能才在推动 人类使生活成为“真正的人的、值得过的”生活的进程中, 发挥了有限却又重要的创造文化发展条件的作用, 做出了 不断造福人类的伟大贡献。

五千多年的中华文明对器的作用的认识早于西方。 《论语-为政》说到“子曰: 君子不器”。何谓君子不器? 就 是说, 仁义之人 (君子), 是不会满足器具利益的获取 (不 器)。另一层意思又有位尊之人 (君子), 是不屑器具的 雕虫小技的 (不器)。显见, 后者又体现了轻视工匠及工 艺的消极态度。儒家的这些精神所代表的中华传统伦理文 化也自然地渗透在我国传统医药学的精义之中。

由上看来, 古今中外都十分强调医学的科学文明的本 质, 为使人类能够有尊严的、有价值的生活进程多元化地 提供了创造文化发展条件的重要作用。这就提醒我们在任 何时候医学研究和临床应用都不能重器而亡义。显见, 基 因组编辑婴儿事件对基因工程先进技术有效性的非理性 追求而忽视了结果可能对社会文化生态造成的深远影响, 就是对人类已经形成共识的基本医学精神的背叛。

\section{3. 事件对现行法规的触犯}

公民对医生的职业充满了敬畏, 同时社会也充分了解 医生决定权被滥用的危害, 为此, 必须要有一种社会公权 力的体现方式来保护病人和规范制约医生。古巴比伦的
《汉谟拉比法典》被认为是世界上第一部较为完善的法典, 就有关于外科医生的条款; 以后法律的完善又增添了保护 医生的相应条文。尽管John D.Bransford[2]说过“法律和规 则只代表我们日常生活中受到别人对解决同样问题影响 和制约的小部分”。（Laws and rules represent only a small part of our everyday lives affected by other people's solutions to problems.) 我们依然需要对现行法规有所敬畏, 在实践 中严格执行不得违背。同时, 我们也需要充分意识到个人 行为是有受同时代、同地域人群及社会主流价值观评判和 监督的义务。而这种道德伦理的自觉服从可能要比法律的 遵从更具普遍性, 更有影响力。

当前, 在美国、欧洲、日本等绝大部分科学技术发达 国家, 是严格禁止编辑人类胚胎的, 违法进行编辑人类基 因组的甚至要受到法律的制裁。在中国开展以生殖为目的 的人类胚胎基因编辑临床操作也是被明令禁止的。基因组 编辑婴儿出生事件显然打破了世界范围内关于“基因编辑 胚胎不得用于妊娠目的”的现行法理的共识。美国哈佛医 学院著名干细胞生物学家乔治·戴利 (George Daley) 11月 28 日就在世界第二届人类基因编辑峰会上发表演讲说到 [3]: 随着科学技术的发展, 一些基于当时发展水平制定的 准则总要被重新讨论, 目前临床医学在基因治疗上还缺乏 对最优方法的共识, 因此需要讨论制定一条清晰的发展 “路线图”, 而其中“允许与不允许 (Permissibility) ”将是 重要的衡量标准。对于科学家来讲, 总会有争做先锋的“诱 惑”。长期以来做研究与临床的群体, 都遵循着“自我管理 /自我监督（Self-regulation）”的原则, 这也是医学界的专 业操守之一。

这充分表明, 基因组编辑婴儿事件不仅触犯了不得将 编辑基因组技术用于人类胚胎的医学研究和临床操作的 不允许触碰的法理底线, 也严重地违背了国内外医学界达 到共识的专业操守。事件还提醒我们需要加强对科学工作 者如何正确地认识和处理科学技术创新与争做先锋诱惑 的利益权衡, 以及一些基于当时发展水平制定的准则及时 调整等重大问题的关注和研究。

\section{4. 事件对伦理规范的挑战}

科学技术研究活动必须要有所为, 有所不为; 任何一 项缺乏法理和伦理规则约束的科学技术实践, 都有可能带 来不可预测的恶果, 而违背科学研究的初衷。越来越多的 学者接受了医学并不是简单的自然科学, 而是自然科学、 社会科学及人文科学综合而成的“人学”的观念, 因此与医 学相关的实践活动与一般的工程技术相比, 更加需要法律 法规和伦理规范的指导和自我制约。医学涉及到的是最基 本的人权保障和健康教育领域的核心问题。伦理规范是在 生物医学研究领域的一类十分广泛且又重要的规范, 尤其 在涉及对人体的研究中体现得更多。其中较为重要的包括 对人实施实验的必要性、实施过程中对人知情权和健康权 的尊重, 以及对实验后果的充分评估等方面。就科学和伦 理的整体功能的理性比较而言, 前者的理性属于一种工具 的理性, 它所追求的目标是客观真理的完善; 而后者的理 性属于价值的理性, 追求的是人类权益的公平。二者具有 相辅相成、相互制约的作用。孙福川教授[4]在《医学伦理 
学》（第四版）的绪论中指出, “在古今中外, 若没有伦 理, 医学的产生和发展是不可想象的。医学尘须有伦理的 支持是一个古老而又常新的命题; 医学必须有伦理的调控, 这是一个格外令人关注的现代命题”。

“第二届人类基因组编辑国际峰会组委会声明”已经 指出了事件在伦理学方面的具体缺陷 [5]: “我们建议进行 独立评估以验证此事, 并确定所声称的DNA修饰是否已发 生。即使基因修改得到验证, 该试验也是不负责任的, 不 符合国际规范, 其缺陷包括医学适应症不足, 研究方案设 计不合理, 未能达到保护研究对象福利的伦理标准, 且在 临床试验的制定、审查和实施中缺乏透明度”。

可以看出, 基因组编辑婴儿事件不仅没能对人实施实 验的必要性、实施过程中对人知情权和健康权的尊重, 以 及对实验后果的充分评估等方面做足功课, 而且即使是没 有出现技术本身的移码突变和完全脱靶等重大问题, 也存 在着不符合国际规范和伦理标准。甚至主持试验者在得知 过程中已经出现不符合条件的测序结果的情况下, 仍然进 行胚胎移植的非理性医学行为。更为严重的是, 事件的主 要负责群体, 置从事此项研究和医学实践危险性的科学预 警于不顾, 直接违背了科学伦理的最基本原则。早在计划 实施前的2016年[6], 美国国家情报总监James R. Clapper 就将基因组编辑列为一种潜在的大规模毁灭性武器

(named genome editing as a potential weapon of mass destruction）, 预见性地指出进行基因组编辑可能增加制 造有害生物制剂或产品的风险。其蓄意或无意的滥用可能 会对经济和国家安全产生深远的影响。诺菲尔德生物伦理 委员会（Nuffield Council on Bioethics）在9月的一份报告 也指出, 修改一个人的基因组的风险和好处以及将这些改 变传给后代是如此复杂的一件事, 急待伦理审查 (demand urgent ethical scrutiny）。这些改变可能会产生意想不到的 后果, 不仅会伤害孩子, 而且因为改变的基因将会存在于 他们的精子或卵子中而伤害到他们未来的孩子。可以看出, 科学家和医学界之所以没有去抢这个世界第一的历史性 突破, 并不是他们技术不过硬, 而是出于他们不能和不愿 去冒可能会危及国家安全和民族延续风险的科学家良知!

在 2018 GES教育未来大会的“教育+科技的伦理与边 界”主题圆桌讨论中, 西安交通大学副校长郑庆华教授谈 到[7], “我们要让科学技术真正服务于教育人, 引导人, 引领人这个方向发展”。“要在这个基础上更多地去分析伦 理、隐私等等这些问题对我们教育的影响, 从而做到有选 择地发挥科学技术作用”。这就从更加广泛的范围和社会 意义上强调了人类应该有选择地发挥科学技术的作用以 及医学伦理的教育功能发挥的问题。特别是对于一些重大 的科学技术突破所带来的利益红利, 必须要从国家层面上 考虑到全局性的发展格局, 而不能急功近利。

\section{5. 事件的理性评价和反思}

任何历史的重大事件的发生都有其必然性和偶然性, 也有其带有时代特征和发展阶段性的积极价值和消极影 响。这在科学技术发展史和医学史上都不乏实证的例子。 不少专家学者都认为, 基因组编辑贞儿事件虽是一个科学
研究与临床实践的阶段性孤例, 却不是一件孤立的事件。 贺建奎研究团队研究工作的技术基础基因组编辑是有科 学依据的, 也是有着可预见的发展潜力的, 是值得研究探 索的; 研究过程中出现技术失误是正常的、多见的, 是科 学探索过程中结出硕果的必要付出。问题主要不是出自技 术层面, 而是精神层面。无论是科学研究实践还是对科学 实践成果的评价, 都不能把洗澡水和婴儿一块泼出去。在 信息时代如何适时地给出正确的评价和导向, 有效地消除 消极影响并积极地去做转化工作, 引导理性地、有选择地 发挥科学技术作用是学术界特别是医学伦理学界一项十 分重要的任务。

简单地说, 伦理是指人际之间符合某种道德标准的行 为准则。对于基因组编辑贞儿事件的伦理评价和反思首先 应该聚焦于社会对事件相关人的道德准则的适度把握和 实施。具体来说就是如何客观而公正地评价贺建奎研究团 队的功过以及对露露和娜娜姐妹社会角色的合乎伦理的 预设。

人们忧虑地看到: 事件发生后出现的一些比较极端的 主张, 尽管是少数但却不缺少市场。比如, 有媒体引用“这 一事件所作的人体实验是极其不人道、反伦理的, 可以视 为反人类罪”的提法。我们不得不指出, 这个命题的结论 是存在法理和政治错误的误判。首先，2002年7月1日生效 的《国际刑事法院罗马规约》[8]中危害人类罪 (即所说的 反人类罪）的定义“是指那些针对人性尊严及其严重的侵 犯与凌辱的众多行为构成的事实。这些一般不是孤立或偶 发的事件, 或是出于政府的政策, 或是实施了一系列被政 府允许的暴行”。其次, 我国的法律体系是不存在这一罪 名的。理性的批判是不能脱离批判的理性的。对于如何对 待犯了过错的科学工作者和医学工作者, 我们十分赞同任 正非老总2018年9月29日在华为公司内部的公共关系战略 纲要汇报会上那句有远见卓识的话[9]“我们要宽容探索创 新的科学家”。宽容是中华民族的传统美德, 也是伦理的 基本内涵之一。一个文明社会要宽容在科学技术探索过程 中的失误和失败, 也要宽容那些甚至触犯了法律和伦理底 线而能吸取教训坚持继续探索创新的那些人。要懂得文明 需要宽容, 科学需要宽容, 社会的发展也需要宽容。

另外一个问题是有关露露和娜娜参与研究过程的选 择权问题。有这样的观点认为: “露露和娜娜的生命不属 于贺建奎的实验团队, 也不单属于孩子的父母, 同时也更 属于全人类。这次事件一定会写进人类的历史。但这一切 的出发点, 是让两个新生命能够正常、健康地生活。而不 是去监视或者管控她们, 更不要妄想去随意剥夺她们的生 命”。这种观点是建立在对生命权的一种好心的误解基础 上的。生命是人格的载体; 生命权是自然人的一项根本的 人格权, 它的主体和客体均为人自身, 法律不允许把生命 直接作为实现任何进一步目的之途径。露露和娜娜作为自 然人已经拥有自己的生命权, 不属于她以外的任何个人 （包括父母）和团体, 更不要说属于全人类。她们还会受 到不能被用于进一步的试验目的的法律保护。《纽伦堡法 典》[10]还清楚地表明“当受试者在实验过程中, 已经达到 这样的肉体和精神状态, 即继续进行已不可能的时候, 完 全有停止试验的自由”。我们认为, 应该立即终止把露露 和娜娜看作是“实验对象”的特殊人的状况, 整个社会要尽 
量让她们过着和大家一样的“真正的人的、值得过的”普通 生活, 更不要再去打设计、掌控她们未来的任何主意!

在贺建奎事件发生之前, 我国生命科学和医疗卫生学 界就已经表现出对此项技术应用于临床的关注和基本倾 向, 以及多角度视点之间的分歧。比如, 《生命科学》2018 年的9月出版了基因编辑技术 2.0 专刊, 其中论文[11-12]分 别从基础研究和临床应用探讨了涉及应用于人类生殖的 界限以及制约因素等问题。《医学与哲学 $(A) 》 2016$ 年以 专论的显著位置发表了邱仁宗的从伦理学的视角对涉及 人类生殖的临床研究工作开展的相关社会对策问题的论 文[13]。遗憾的是却未能阻止事件的发生, 不过庆幸的是 事件的出现更加促进了人们对基因组编辑技术研究和应 用的理性思考和科学实践。

\section{6. 结语}

基因组编辑婴儿事件并未终结, 而且还会受到社会和 伦理学界的继续关注。它给了科学工作者不少启发和警示, 促进科学界的理性反思和探究。在科学技术高速发展、创 新已经成为社会发展重要引擎的新时代, 人类社会更加需 要发挥伦理学对医学的支持和调控作用, 促进医学的健康 有序发展和有选择地发挥科学技术作用, 强化医学事业的 内部自律和医学研究和临床工作者坚持操守、克服争做先 锋的诱惑, 推动医学不断对人类福祉做出更大贡献, 为人 类社会的发展创造更扎实的文化发展条件。

\section{参考文献}

[1] 亨利-欧内斯特-西格里斯特.疾病与人类文明 $[\mathrm{M}]$. 北京: 中 央编译出版社, 2016: 289。

[2] John D.Bransford, Barry S. Stein. The Ideal Problem Solver [M]. New York: W. H. Freeman and Company, 1984:
［3］徐路易.哈佛生物学家：希望基因编辑技术的发展不受贺建 奎事件影

响.http://news.sina.com.cn/o/2018-11-28/doc-ihmutuec43459 97.shtml

[4] 孙福川, 王明旭主编. 《医学伦理学》 (第四版) [M], 北 京：人民卫生出版社，2013：2-3。

[5] 峰会大会组委会. 第二届人类基因组编辑国际峰会组委会 声明 (叶水送, 邸利会 译)

https://mp.weixin.qq.com/s/MwcUC9clsOFpFuZ8BW43Yw, $2018,11,29$.

[6] Wikipedia: genome editing, https: //en.wikipedia.org /wiki/ Genome_editing, edited on 7 Dec. 2018.

[7] Pamela: 教育+科技的伦理与边界: 科技技术的双面性(鲸媒 体2018-12-05 ), http://www.sohu.com/a/279727329_361784.

[8] 百度百科: 反人类罪. https://baike.baidu.com/item/危害人类 罪/4405861?Fromtitle http://xinsheng.huawei.com/cn/index.php?app=forum\&mod= Detail\&act $=$ index\&id $=4092231$

[9] 任正非.从人类文明的结晶中, 找到解决世界问题的钥匙 一任总在公共关系战略纲要汇报会上的讲话(公司文件). 2018年9月29日。

[10] 王德国. 探讨《纽伦堡法典》中人体实验的伦理原则与规 范 $[J]$. 中国医学伦理学, 2016, 29 (2) : 311。

[11] 张晓宇, 唐蔚, 李劲松. CRISPR/Cas9系统应用于早期胚胎 编辑和基因治疗 [J]. 生命科学, 2018, 09:0916-0925。

[12] 李广磊, 曾艳婷, 刘见桥. 基因编辑技术在人类生殖细胞中 的应用研究 [J]. 生命科学,2018,09:932-939。

[13] 邱仁宗. 基因编辑技术的研究和应用:伦理学的视角 [J]. 医 学与哲学 (A)，2016,07A:01-07。 